\title{
Liraglutide increases IVF pregnancy rates in obese PCOS women with poor response to first-line reproductive treatments: a pilot randomized study
}

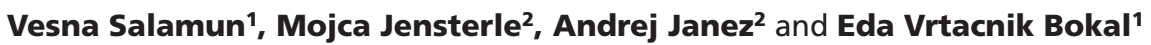 \\ 'Division of Obstetrics and Gynecology, Department of Human Reproduction and ${ }^{2}$ Department of Endocrinology, \\ Diabetes and Metabolic Diseases, University Medical Centre Ljubljana, Ljubljana, Slovenia \\ Correspondence \\ should be addressed \\ to V Salamun \\ Email \\ vesna.j.salamun@gmail.com
}

\begin{abstract}
Objective: Glucagon-like peptide-1 (GLP-1) has been investigated in regulation of reproductive system in animal models. The potential impact of short-term preconception intervention with liraglutide on fertility potential in polycystic ovary syndrome (PCOS) has not been evaluated yet.

Design: A prospective randomized open-label study was conducted in 28 infertile obese PCOS patients (age: $31.07 \pm 4.75$, BMI: $36.7 \pm 3.5 \mathrm{~kg} / \mathrm{m}^{2}$, mean \pm s.D.). They were assigned to metformin (MET) $1000 \mathrm{mg}$ BID or to MET $1000 \mathrm{mg}$ BID combined with low-dose liraglutide $1.2 \mathrm{mg}$ QD s.c. (COMBI) for 12 weeks. Ovarian stimulation protocol was started after a 4-week medication-free period.

Methods: The in vitro fertilization pregnancy rate (PR) was defined as the number of clinical pregnancies confirmed by ultrasound visualization of the fetal cardiac activity, divided by the total number of cycles performed or embryo transfers (ET). The spontaneous PR was followed for 12 months.

Results: Patients in the MET group on average lost $7.0 \pm 6.0 \mathrm{~kg}(P=0.001)$ compared with $7.5 \pm 3.9 \mathrm{~kg}$ in the COMBI group $(P<0.001)$ with no significant between-treatment difference $(P=0.246)$. The PR per ET was significantly higher in the COMBI $(85.7 \%)$ compared with the MET $(28.6 \%)$ group $(P=0.03)$. The cumulative PR in the time frame of 12 months was $69.2 \%$ in the COMBI group compared to $35.7 \%$ in the MET group.

Conclusions: Preconception intervention with low-dose liraglutide added to metformin is superior to metformin alone in increasing PRs per ET and cumulative PRs in infertile obese women with PCOS, despite comparable weight reduction in both groups. A potential impact of liraglutide on the reproductive system needs further exploration, in particular the GLP-1 impact on endometrial quality and receptivity.
\end{abstract}

\section{Introduction}

Polycystic ovary syndrome (PCOS) is the most common cause of anovulatory infertility and affects up to $20 \%$ of women of reproductive age according to the Rotterdam criteria $(1,2)$. Nearly half of the women with PCOS are overweight or obese (3). The impact of obesity on in vitro
European Journal of Endocrinology (2018) 179, 1-11 
Weight loss prior to IVF procedures has been associated with significantly improved pregnancy rates (PR) and live birth rates $(7,8,9,10)$. Furthermore, a decreased number of IVF cycles required to achieve a pregnancy has also been reported after weight loss interventions (11). Based on the principles of fetal programming, improving a lifestyle before conception might lead to improved longterm health of the offspring $(12,13)$.

Lifestyle modification is recommended as the firstline treatment in obese infertile PCOS women $(14,15)$, whereas clomiphene citrate (CC) remains the secondline treatment for ovulation induction. Compared to CC, metformin is inferior in improving the respective rates of ovulation, pregnancy, live birth, miscarriage and multiple pregnancy (16). A potential benefit of metformin has been found in women with BMI greater than $35 \mathrm{~kg} / \mathrm{m}^{2}$ when combined with CC (17).

Long-acting glucagon-like peptide-1 (GLP-1) receptor agonist (RA) liraglutide has provided positive effects on weight reduction and glucose homeostasis in obese PCOS women $(18,19)$. It provides meaningful weight loss when combined with metformin compared to metformin only and lifestyle modification $(18,20)$. The mechanism of body weight reduction appears to be due to decreased food intake, mainly by a direct hypothalamic effect. It also delays gastric emptying partly due to a central action mediated via the autonomous nervous system $(21,22)$.

The potentially beneficial effects of liraglutide on IVF outcomes of PCOS obese patients have not been addressed yet. Since liraglutide is an effective insulinsensitizing agent there is a theoretical assumption that weight loss will improve the reproductive outcomes by weight loss. Moreover, new animal data suggest that GLP-1 agonist plays a role in the regulation of hypothalamus-pituitary-ovary axis by neuroendocrine modulation of gonadotropin-releasing hormone release (23).

The aim of this pilot study was to evaluate the impact of low-dose liraglutide in combination with metformin compared to metformin alone on IVF PRs and cumulative PRs (IVF and spontaneous pregnancies) in infertile obese women with PCOS who had been previously poor responders to weight reduction with lifestyle modification, and resistant to the first-line reproductive treatments with CC or aromatase inhibitors. The secondary outcomes were to evaluate the impact of therapy on oocyte and embryo quality, weight loss and metabolic and endocrine parameters.

\section{Subjects and methods}

\section{Subjects}

Twenty-eight obese PCOS patients diagnosed according to the revised Rotterdam criteria (2) participated in a 12-week open-label prospective randomized outpatient clinical study between September 2014 and May 2015 . Eleven women receiving the combined therapy and 8 receiving metformin had the classic $\mathrm{PHO}$ phenotye (PCO morphology $(\mathrm{P})$, hyperandrogenic features $(\mathrm{H})$ and oligoamenorrhea $(\mathrm{O})$ ). Two women receiving metformin had the $\mathrm{PH}$ phenotype, and one had the PO phenotype. Two women receiving the combined therapy and 3 receiving metformin had the HO phenotype (24). The inclusion criteria were as follows: PCOS diagnosed according to the revised Rotterdam criteria, body mass index (BMI) $\geq 30 \mathrm{~kg} /$ $\mathrm{m}^{2}$, age $\leq 38$, first or second IVF attempt; no severe male infertility. The exclusion criteria were type 1 or type 2 diabetes mellitus, history of carcinoma, personal or family history of MEN2, significant cardiovascular, kidney, or liver disease, use of medications other than metformin known or suspected to affect reproductive or metabolic functions or statins within 90 days before study entry, and no coexisting ovarian pathology.

All patients were informed of the study aims and signed the written consent form before entering the study, which was conducted in accordance with the Declaration of Helsinki and approved by the National Medical Ethics Committee (approval number: 161/08/14). The study is registered with ClinicalTrials.gov identifier: NCT03353948.

\section{Study protocol}

Patients were randomized to one of the two treatment arms: metformin (MET) $1000 \mathrm{mg}$ BID or metformin $1000 \mathrm{mg}$ BID combined with $1.2 \mathrm{mg}$ liraglutide QD s.c. (COMBI). Metformin was initiated with a dose of $500 \mathrm{mg}$ once per day and increased by $500 \mathrm{mg}$ every 3 days up to $1000 \mathrm{mg}$ BID. In the COMBI arm there was a run-in period of 12 days to titrate metformin up to $1000 \mathrm{mg}$ BID before liraglutide was added. Liraglutide was initiated with a dose of $0.6 \mathrm{mg}$ injected s.c. once per day and increased to $1.2 \mathrm{mg}$ after 3 days. Medical treatment in both groups lasted 12 weeks.

At the beginning of the study, lifestyle intervention was actively promoted in both groups. A reducing diet of $500-800 \mathrm{kcal} /$ day consisting of up to $50 \%$ of carbohydrates preferably with low glycemic index, $20 \%$ of proteins and 
$30 \%$ of fat, mostly mono- and poly-unsaturated, with the amount of saturated fat less than $10 \%$, was advised. The participants were encouraged to increase consumption of fibers, whole grains, cereals, fruits and vegetables along with at least $30 \mathrm{~min}$ of moderate-intensity physical activity daily. The IVF procedure was offered to all the patients that completed the medical treatment after 1 month of medicine-free period. At baseline and at study end fasting blood was drawn for determination of glucose, insulin, androstenedione, total and free testosterone, sex hormone-binding globulin (SHBG) and safety parameters (complete blood count, markers of hepatic and renal functions). The standard 75-g oral glucose tolerance test (OGTT) was performed (25). We informed the women about the eventual unknown effects of liraglutide, and recommended barrier contraceptives according to the personal preferences of the women.

\section{Assessment of anthropometric parameters}

All patients underwent clinical, anthropometric and biochemical assessment at the baseline and at study endpoint. Waist circumference was measured in a standing position midway between the lower costal margin and the iliac crest. BMI was calculated as the weight in kilograms divided by square of height in meters. Whole-body composition was carried out on a Hologic dual energy X-ray absorptiometry (DXA) (Discovery A) with the software provided by the manufacturer (QDR for Windows, Version 12.5) (26).

\section{Assessment of metabolic parameters}

Glucose levels were determined using the standard glucose oxidase method (Beckman Coulter Glucose Analyzer, Beckman Coulter Inc., CA, USA). Insulin was determined by immunoradiometric assay (Biosource Europe S.A., Nivelles, Belgium). Insulin resistance (IR) was calculated by the homeostasis model assessment for IR (HOMA-IR): fasting serum insulin $(\mathrm{U} / \mathrm{L}) \times$ fasting plasma glucose $(\mathrm{mmol} / \mathrm{L}) / 22.5$ (27). Impaired glucose tolerance (IGT) was identified by 2-hour glucose levels between 7.8 and $11.0 \mathrm{mmol} / \mathrm{L}$, as defined by the American Diabetes Association criteria (25).

\section{Assessment of endocrine parameters}

Androstenedione was measured by specific double antibody RIA using 125 I-labeled hormones (Diagnostic
Systems Laboratories, Webster, TX). Total and free testosterone levels were measured by coated tube RIA (DiaSorin, S. p. A., Salluggia, Italy and Diagnostic Products Corporation, LA respectively). Intraassay variations ranged from 1.6 to $6.3 \%$, and interassay variations ranged from 5.8 to $9.6 \%$ for the applied methods. Pre- and posttreatment samples for each patient were assayed in the same assay run.

\section{Ovarian stimulation protocol}

The short protocol of GnRH antagonist cetrorelix was used for ovarian stimulation in all the patients. The protocol consisted of daily stimulation with $225 \mathrm{IU}$ of follicle stimulating hormone (Puregon; ScheringPlough, Kenilworth, New Jersey, USA) from day 2 of the menstrual cycle. GnRH antagonist cetrorelix acetate (Cetrotide; Merck Serono) in a dose of $0.25 \mathrm{mg}$ s.c. was administered when the dominant follicle measured $14 \mathrm{~mm}$ in diameter. In all the patients buserelin acetate $0.6 \mathrm{mg}$ s.c. (Suprefact; Hoechst AG, Frankfurt/Main, Germany) was administered for oocyte maturation when at least three dominant follicles measured $\geq 17 \mathrm{~mm}$ in diameter.

\section{Oocyte retrieval, fertilization, embryo culture and transfer, luteal support}

Transvaginal ultrasound-guided oocyte retrieval was performed under local anesthesia $36 \mathrm{~h}$ after buserelin administration. Oocytes were fertilized by intracytoplasmic sperm injection (ICSI). Embryos were cultured to the blastocyst stage in sequential media M1 and M2 (Origio; MediCult, Jyllinge, Denmark) until day 5. One or 2 best quality blastocysts were transferred into the uterus on day 5. If fewer than 3 oocytes were retrieved, the embryos were transferred on day 3. In case of supernumerary blastocysts or at increased risk of ovarian hyperstimulation syndrome blastocysts were frozen on day 5 or day 6 using a modified two-step Menezo method (28) and stored in liquid nitrogen at $-196^{\circ} \mathrm{C}$.

Luteal phase support consisted of $1500 \mathrm{IU}$ of human chorionic gonadotropin (hCG) (Pregnyl; N.V. Organon, Oss, The Netherlands) administered after oocyte retrieval if fewer than 21 oocytes were retrieved. Progesterone (Estima Ge; Laboratoires EFFIK, Bruxelles, Belgium) was given intravaginally in a daily dose of $3 \times 200 \mathrm{mg}$ until a gestational sac with fetal cardiac activity was visible on day 30 after the ET. 


\section{Assessment of reproductive parameters}

Oocyte maturity (MII stage) was defined as germinal vesicle break down (GVBD) with the presence of a polar body. Oocytes with a germinal vesicle or GVBD without a polar body (MI stage) were considered immature. Fertilization rate was defined as the ratio between the number of fertilized and mature oocytes. Oocyte degeneration rate was defined as the ratio between the number of degenerated and all retrieved oocytes. Immaturity rate was defined as the ratio between the number of immature and all retrieved oocytes. Blastulation rate was defined as the ratio between the number of blastocysts and mature oocytes. The blastocyst grading system introduced by Gardner et al. was used to evaluate the blastocyst quality (29).

Pregnancies were diagnosed by beta hCG serum determination 14 days after hCG administration and by a transvaginal ultrasound examination 4 weeks after the ET. PR was defined as the number of clinical pregnancies (ultrasound visualization of fetal cardiac activity) divided by the total number of cycles performed or embryo transfers. Cumulative PR was defined as the number of all pregnancies (spontaneous and IVF) divided by the number of patients. The spontaneous pregnancy rate was followed for 12 months after the end of antiobesity intervention.

\section{Statistical analysis}

The results are presented as means \pm S.D. or percentages as appropriate. Normal data distribution was checked with the Shapiro-Wilk test. For the normally distributed data, $t$-test was used (paired samples $t$-test for comparison of before/after parameters, independent samples $t$-test for comparison between the arms). For non-normally distributed data Mann-Whitney $U$ test and Wilcoxon signed ranks test were used. For categorical data we used chi-square test. $P$ value of 0.05 was considered statistically significant. Statistical analysis was performed using IBM SPSS Statistics, version 24 (IBM Corp, Armonk, NY).

\section{Results}

\section{Population characteristics}

Twenty-eight patients started the study, and 27 (14 on MET, 13 on COMBI) completed the treatment according to the protocol and were included in the analysis (Fig. 1). One patient in the COMBI group discontinued the study because of protocol violation, 2 patients in the MET group refused IVF. One patient in the COMBI and 1 in the MET group conceived spontaneously immediately after the completed treatment. The remaining 23 patients (11 in the MET and 12 in the COMBI group) attended an IVF after the completed treatment.

\section{Baseline results}

There were no significant differences in patients' characteristics of infertility history between the treatment groups (Table 1). There were no significant differences at baseline in any of the anthropometric, metabolic and endocrine parameters between the groups except in 120 min overload insulin levels (Table 2).

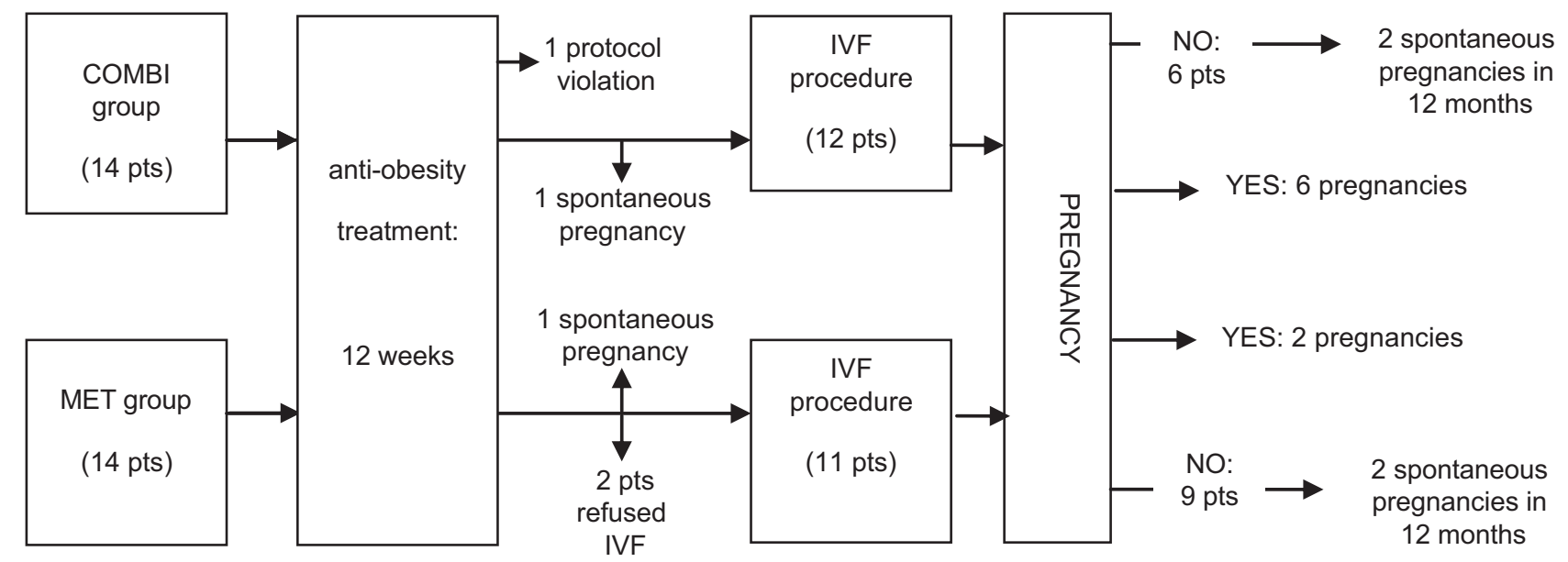

Figure 1

Flowchart of the study with included pregnancies during the observation time of 1 year. COMBI, combination of metformin and liraglutide treatment arm; IVF, in vitro fertilization; MET, metformin treatment arm; pts, patients. 
Table 1 Data of infertility history. Values are mean \pm s.D. or $\%$.

\begin{tabular}{|c|c|c|c|}
\hline & MET $(n=14)$ & COMBI $(n=13)$ & $P$ value \\
\hline Age (years) & $31.1 \pm 4.7$ & $30.1 \pm 3.6$ & NS \\
\hline Infertility duration (month) & $32.7 \pm 20.1$ & $56.2 \pm 33.3$ & NS \\
\hline Primary type of infertility (\%) & $50.0(7 / 14)$ & $75.0(9 / 13)$ & NS \\
\hline Unsuccessful attempt to reduce weight (\%) & $78.6(11 / 14)$ & $75.0(9 / 13)$ & NS \\
\hline Previous therapy: CC (\%) & $50.0(7 / 14)$ & $61.5(8 / 13)$ & NS \\
\hline Previous therapy: AI (\%) & $21.4(3 / 14)$ & $30.8(4 / 13)$ & NS \\
\hline LEKO (\%) & $28.6(4 / 14)$ & $53.8(7 / 13)$ & NS \\
\hline
\end{tabular}

$\mathrm{Al}$, aromatase inhibitors; CC, clomiphene citrate; COMBI, combination of metformin and liraglutide treatment arm; LEKO, laparoscopic electrocoagulation of ovary; MET, metformin treatment arm; NS, not significant.

\section{Changes in measures of obesity}

Body mass decreased significantly in both treatment groups $(P=0.001$ in the MET, $P<0.001$ in the COMBI group). Weight decreased similarly in the MET and the COMBI group $(P=0.246)$. Of the 27 patients, 17 $(63.0 \%)$ were high responders who lost more than $5 \%$ of body weight in 3 months $(69.2 \%(9 / 13)$ in the COMBI vs $57.1 \%(8 / 14)$ in the MET group). Compared to the baseline, the patients in the MET and the COMBI group lost on average $6.99 \pm 6.02 \mathrm{~kg}$ and $7.51 \pm 3.89 \mathrm{~kg}$ respectively. No patient experienced a net weight gain. The data of the post treatment body mass is presented in Table 2.

Likewise, a significant BMI reduction of $2.5 \pm 2.0 \mathrm{~kg} /$ $\mathrm{m}^{2}$ in the MET, and $2.7 \pm 1.3 \mathrm{~kg} / \mathrm{m}^{2}$ in the COMBI group was noted after treatment compared to the baseline $\left(P^{<} 0.001\right.$ in the MET and the COMBI treatment) with no difference in the treatment employed $(P=0.791)$.

Comparable results were obtained for the body waist circumference with a significant reduction in the treatment/time effect in both therapeutic groups. The patients treated with MET lost on average $11.3 \pm 9.2 \mathrm{~cm}$ and the patients on COMBI lost $11.7 \pm 9.0 \mathrm{~cm}(P=0.001$ in the MET and COMBI group) of waistline. All treatment interventions resulted in significant reduction of visceral adipose tissue mass, volume and area as assessed by DXA. At 3 months, the average visceral adipose tissue (VAT) area in both groups decreased below $160 \mathrm{~cm}^{2}$, the threshold associated with a high risk of cardiovascular disease in women (30).

\section{Metabolic changes}

Seven ( 2 in the MET, 5 in the COMBI group) patients had IGT at the beginning of the study. After the treatment, 3 patients in the COMBI and none in the MET group normalized their response to the glucose upload.
HOMA-IR score values were reduced after both interventions, the reduction being significant only in the COMBI group $(P=0.04)$. Fasting and post OGTT decreased significantly also in the COMBI group only $(P=0.004$ and $P=0.05$ respectively) (Table 2 ).

\section{Endocrine changes}

There was a significant increase in SHBG noted in the MET and COMBI treatment arm compared to the baseline (MET: $P=0.019$, COMBI: $P=0.03$ ) (Table 2 ).

\section{Pregnancies and IVF parameters}

In the COMBI group, 9 of the $13(69.2 \%)$ patients became pregnant in one year. One patient conceived spontaneously immediately after treatment, 6 pregnancies occurred after an IVF procedure and 2 spontaneous after an unsuccessful IVF (Fig. 1). In the MET group 5 of the 14 (35.7\%) patients became pregnant in one year: 1 immediately after the treatment, 2 after an IVF procedure and 2 spontaneous after an unsuccessful IVF. Two patients in the MET group declined IVF for personal reasons (Fig. 1).

The PR per ET was significantly higher in the COMBI (85.7\%) compared to the MET group (28.6\%) ( $P=0.03)$. There was no statistically significant difference between the groups by the dose of stimulation, number of retrieved oocytes, respective number of mature, fertilized and degenerated oocytes, number of embryos and number of blastocysts on day 5 (Table 3).

\section{Adverse events}

The adverse events were transient and mild. The patients in the MET group reported nausea (1/14) and diarrhea $(1 / 14)$. The patients in the COMBI group reported nausea $(5 / 13)$ and headache $(2 / 13)$. Low-dose and gradual titration reduced the GI side effects associated with liraglutide. 


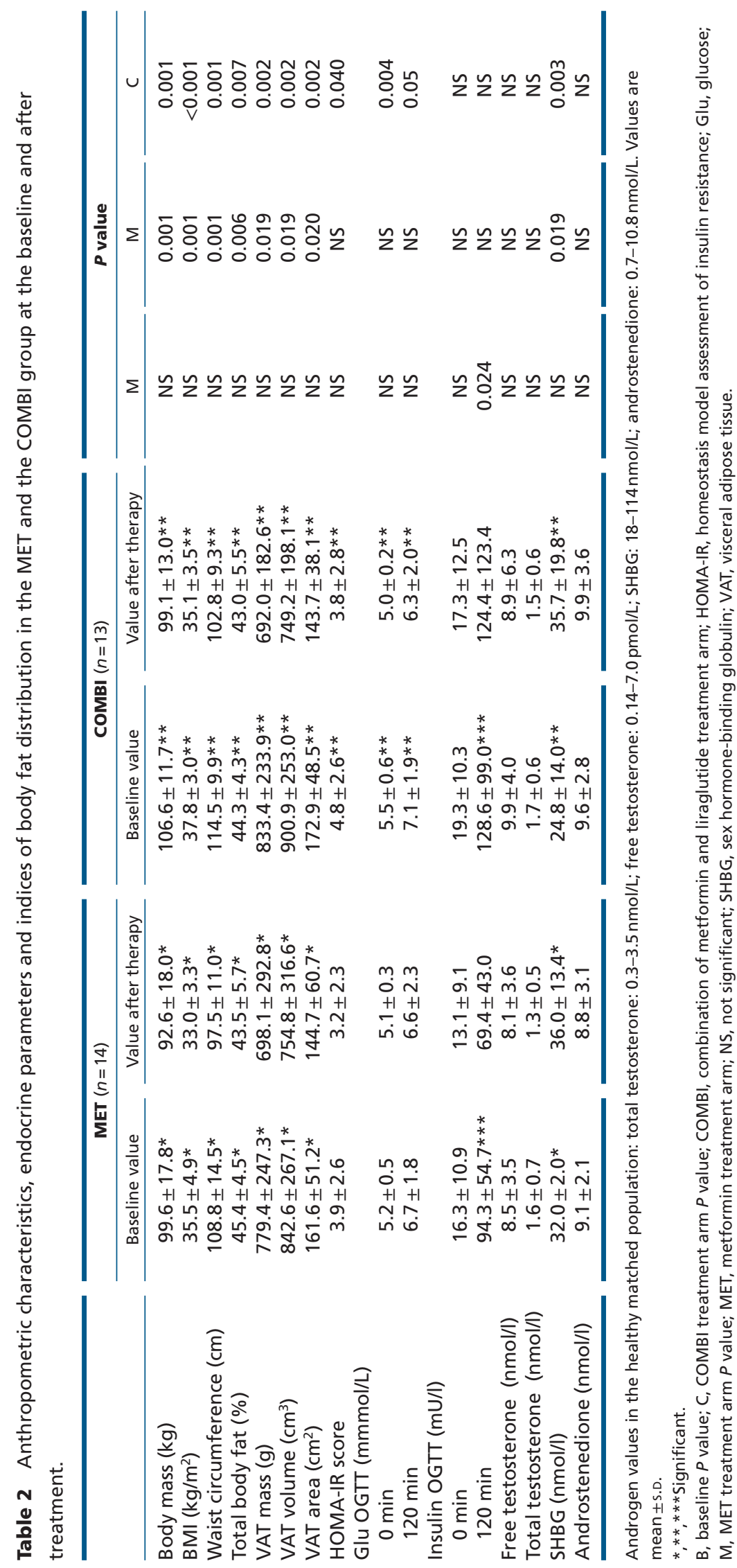


Table 3 IVF outcomes in the MET and the COMBI group.

Total dosage GNT (IU)
No. of retrieved oocytes/patient
No. of mature (MII) oocytes/patient
Fertilization rate $(\%)$
Oocyte degeneration rate (\%)
Immaturity rate (\%)
No. of embryos on day $5 /$ patient
No. of blastocysts/patient
Blastulation rate $(\%)$
No. of transferred embryos
Pregnancy rate per cycle (\%)
Pregnancy rate per ET (\%)
Implantation rate (\%)
No. of cancelled fresh ET because of hyperstimulation risk
Cryopreservation ( $\%)$
No. of cryopreserved embryos/patient

\begin{tabular}{c}
\hline MET $(n=11)$ \\
\hline $2250 \pm 275$ \\
$9.7 \pm 5.9$ \\
$6.8 \pm 5.7$ \\
$78.7(59 / 75)$ \\
$11.2(12 / 107)$ \\
$14.0(15 / 107)$ \\
$4.5 \pm 5.8$ \\
$2.4 \pm 4.7$ \\
$36.0(27 / 75)$ \\
$1.4 \pm 0.5$ \\
$18.18(2 / 11)$ \\
$28.57(2 / 7)$ \\
$3 / 10$ \\
2 \\
$45.5(5 / 11)$ \\
$4.8 \pm 6.3$
\end{tabular}

\begin{tabular}{c}
\hline COMBI $(n=12)$ \\
\hline $2172 \pm 320$ \\
$12.8 \pm 9.4$ \\
$8.8 \pm 8.0$ \\
$73.6(78 / 106)$ \\
$10.4(16 / 154)$ \\
$13.0(20 / 154)$ \\
$5.5 \pm 5.5$ \\
$3.2 \pm 4.3$ \\
$35.8(38 / 106)$ \\
$1.3 \pm 0.5$ \\
$50.0(6 / 12)$ \\
$85.7(6 / 7)$ \\
$6 / 13$ \\
4 \\
$58.3(7 / 12)$ \\
$5.0 \pm 4.7$
\end{tabular}

\begin{tabular}{c}
\hline $\boldsymbol{P}$ value \\
\hline NS \\
NS \\
NS \\
NS \\
NS \\
NS \\
NS \\
NS \\
NS \\
NS \\
NS \\
0.03 \\
NS \\
NS \\
NS \\
NS
\end{tabular}

Values are mean \pm s.D. unless otherwise stated.

ET, embryo transfer; MII, metaphase II; NS, not significant.

\section{Discussion}

This is the first report demonstrating that short-term preconception treatment with liraglutide in combination with metformin is superior to metformin alone in increasing PRs per ET and cumulative PRs in obese PCOS women who had been previously resistant to weight reduction with lifestyle modification and firstline reproductive treatments. The observed superiority is challenging, particularly since both treatment interventions resulted in a comparable reduction of body weight and visceral adipose tissue.

Weight loss prior to either spontaneous conception or conception by IVF in obese women is recommended by international guidelines (15), but the data on optimal magnitude and timing are limited. Until now, short-term weight reduction directly before IVF has been assessed in a few prospective randomized controlled studies only ( 7 , $8,9,10,31)$. The results about reproductive outcomes of IVF are inconclusive. Higher PRs and DRs were achieved in weight loss group (48 vs $14 \% ; P=0.007 ; 44$ vs $14 \%$; $P=0.02)$ with diet, exercise and behavioral support (8). A recent Spanish study showed a significantly higher cumulative live birth rate (61.9 vs $30 \%)$ (9). In the study of obese no-PCOS Australian patients who lost their weight of $3.8 \pm 3.0 \mathrm{~kg}$ by diet and physical activity in 5-9 weeks there were no differences in PRs and DRs between the intervention and the control group, the increased odds ratio of pregnancy was associated with a waist circumference reduction (7). By contrast, the largest multicentric study including 290 obese women showed

no improvement in reproductive outcomes due to weight reduction prior to IVF $(31)$.

In the present study, $69 \%$ of women in the COMBI and $57 \%$ in the MET group lost more than 5\% of body weight within a 12-week period of pharmacological intervention along with promoted lifestyle modification. Women in the MET group lost on average $7.0 \pm 6.0 \mathrm{~kg}$, whereas women in the COMBI group lost $7.5 \pm 3.9 \mathrm{~kg}$. Similarly, a significant reduction in BMI, visceral adipose tissue and waist circumference was noted in both treatment groups compared to baseline values/measurements with no differences regarding the type of treatment. The magnitude of weight loss in the COMBI arm was expected and in line with previous reports $(18,19,32)$. On the other hand, the magnitude of weight loss in the MET arm was beyond expectation since the greatest documented absolute weight reduction with metformin in obese women with PCOS is about $2.7 \mathrm{~kg}$ representing less than $5 \%$ of weight reduction (33). Greater weight loss in our cohort might be at least partly explained by the extremely high motivation for lifestyle modification encouraged by a multidisciplinary team of endocrinologists and gynecologists mainly through education about a higher incidence of pregnancy and fetal risks associated with maternal obesity (13).

Within 12 months after a short-term pharmacological intervention prior to IVF, 9 of the 13 (69.2\%) patients in the COMBI and 5 of the $14(35.7 \%)$ patients in the MET group became pregnant. In the COMBI group, 1 pregnancy was spontaneous immediately after treatment, 6 pregnancies were achieved following IVF, and 2 spontaneous after an 
unsuccessful IVF. In the MET group, 1 pregnancy occurred immediately after treatment, 2 following IVF, and 2 spontaneously after an unsuccessful IVF. Improvement in the cumulative PR did not reach statistical significance, but the tendency toward improved fertility potential in the COMBI arm is apparent, particularly if severely resistant infertility of the study population is taken into account. We may speculate that a larger study population would result in a significant improvement in the cumulative PR. The results of our study cannot be directly compared with other prospective randomized controlled studies assessing the impact of short-term weight reduction directly before IVF because there are some substantial differences in outcome measures, study populations and treatment protocols.

The original choice of preconception treatment protocol resulted in a very interesting yet highly challenging observation that the PR per ET was significantly higher in the COMBI (85.7\%) compared to the MET group (28.6\%) despite a comparable weight reduction in both arms. In agreement with the expected PR in PCOS patients attending IVF (around 30\%) we could not prove any additional beneficial effect of metformin on PR per ET (34). By contrast, we noted a highly beneficial effect of liraglutide that increased the PR per ET from the expected $30 \%$ to $85.7 \%$. Furthermore, cumulatively twice as many patients became pregnant using the combination of liraglutide and metformin (70\%) compared to 36\% using only metformin in the observation period of 1 year.

Although the current data on GLP-1 analogs and reproduction are scarce, liraglutide might affect the reproductive system through several different mechanisms. Weight reduction could be one of important mechanisms which was proven in a 24 -week open-label prospective randomized clinical study of 176 overweight/ obese women with PCOS that received short-acting GLP1RA exenatide (Exe) compared to MET. The short-term Exe therapy was linked to significant weight loss $(4.29 \pm 1.29 \mathrm{~kg}$ vs $2.28 \pm 0.55 \mathrm{~kg}, P<0.001)$, total fat percentage reduction, improved IR and increased menstrual frequency ratio. The rate of natural pregnancy in the time frame of 12 weeks in the Exe-treated patients was significantly higher than in the MET-treated patients (43.60 vs $18.70 \%$ ) (35). In general, the proposed mechanism by which weight loss improves menstrual cyclicity and ovulation has been explained mainly by a reduction of visceral fat and improved insulin sensitivity $(36,37)$. Improvement in menstrual frequency addressed as a primary outcome was confirmed in the first study that had used GLP-RA Exe in the PCOS population (38).
The second mechanism of action of liraglutide could be explained by improvement of IR that is associated with weight reduction. The role of IR and hyperinsulinemia in the development of PCOS has been thoroughly explored. It is generally accepted that they play an important role in the pathophysiology of the PCOS and anovulation. Insulin may augment the effects of luteinizing hormone (LH) by increasing the secretion of ovarian androgens (39). Insulin also suppresses the secretion of SHBG by the liver leading to increased levels of free circulating testosterone (39). The improved insulin sensitivity might have the pivotal role in restoring ovarian function. In our study IR as assessed by HOMA-IR score was significantly reduced in the COMBI group. An increase in SHBG that decreased the bioavailability of androgens was also noted in the MET and the COMBI arm. The majority of androgens decreased in both arms, although this decrease did not reach a statistically significant difference.

However, the superior effect of COMBI on the PR per ET despite the comparable reductions of body weight, visceral adipose tissue and androgen bioavailability in both arms imply some unknown effects of liraglutide on fertility potential that is beyond weight reduction and improved IR. Some important animal studies have shown the involvement of GLP-1 in the regulation of the reproductive system through the hypothalamus-pituitary system. Interestingly, intracerebroventricular injection of GLP-1 increases the levels of plasma LH in rats (40). Recently, a study in rats has found that the expression of the GLP receptor varies during the estrous cycle in the hypothalamus, pituitary and ovary. Acute treatment with native GLP-1 during the proestrus phase doubled the amplitude of the LH surge and resulted in increased progesterone in the luteal phase. These changes increased the number of mature graafian follicles resulting in increased fertility. However, treatment with EXE had the opposite effect to GLP-1 reducing the LH surge by 50\% (41). The hypothalamic-pituitary axis is a regulatory system that provides regular menstrual cycles with consequential ovulations, and regulates the feeling of hunger and eating habits. We assume that GLP-1 takes a part in coupling these two activities in the hypothalamus-pituitary system and acts as a metabolic signal to the reproductive system (42, 43). In response to $48 \mathrm{~h}$ of fasting, the rats' hypothalamic GLP-1 content was significantly reduced when there was a rapid reduction in pituitary LH. Upon re-feeding there was a trend for GLP-1 to return to normal, perhaps aiding the recovery of LH secretion (44).

Moreover, from the previous studies we know that diabetes mellitus is associated with severe changes in the 
structure and function of endometrium due to induced cellular toxicity in endometrial epithelial and stromal layers (45). The induced alterations in the endometrium may cause implantation failure, pregnancy loss and defective placentation which have been commonly observed in PCOS as well. In diabetic rats, increased endometrial stromal fibrosis and degeneration were demonstrated. Interestingly, a short acting GLP-1 analog Exe improved the endometrial function in this animal model. The authors suggested that the observed effect of Exe might be mainly due to antagonizing oxidative stress and decreasing transforming growth factor beta levels, thus decreasing fibrosis of the endometrium (46). Since in our study there were no between group differences in the stimulation process (duration, the dosage of gonadotropins), number of oocytes and their quality, number of embryos and their quality, we believe that further research should be focused on the yet unaddressed impact of GLP-1 on the quality and endometrial receptivity.

Regarding the safety issue, GLP-1 RA has a well established favorable 8-year risk-benefit balance in patients with type 2 diabetes and in obese individuals without diabetes (47). The knowledge regarding human exposure to GLP-1 RAs during pregnancy is extremely limited and based on a single reported case of liraglutide exposure up to 13th week of gestation of a woman with type 2 diabetes with a normal pregnancy outcome (48). A preconception intervention with short-term GLP-1 RA Exe has been reported in another case report of an overweight woman with PCOS and IR (49). No important safety issue has been reported in a recent large clinical study evaluating the effects of preconception intervention with Exe on reproductive and metabolic function in overweight/obese PCOS (35). Based on a well established short-term safety profile of liraglutide in patients with type 2 diabetes and obese subjects, and on some limited data on the use of preconception intervention with GLP-1 RAs, a short-term preconception treatment in PCOS subjects seems to be acceptable.

Our study has some limitations. It was conducted as a pilot project and the conclusions are very limited by a small sample size. Further larger studies should be powered using these preliminary results. Spontaneous conception in severely infertile women after weight reduction with liraglutide and metformin encourages the evaluation of potential effects of weight loss on spontaneous conception rates in larger studies with the protocols including delayed IVF procedures in order to avoid unnecessary IVF. Furthermore, the superior impact of COMBI on PRs per ET despite the comparable reduction of body weight in both arms was unexpected, and implies a potential direct crosstalk between GLP-1 and reproductive system that needs further exploration. The results intend to be the basis of more thorough research and proof-ofconcept designs. However, the main strength of this pilot study is the original concept of an efficient short-term weight-reducing intervention with liraglutide directly prior to IVF in infertile obese women with PCOS marked by population and protocol homogeneity.

In conclusion, this is the first pilot study providing preliminary evidence that a short-term preconception intervention with low-dose liraglutide added to metformin is superior to metformin alone in increasing PRs per ET and cumulative PRs in infertile obese women with PCOS despite comparable weight reduction in either treatment. Our observations are not definitive but do encourage further exploration of the efficacy and safety of this class of medications on reproductive outcomes in different obesity-related populations. The precise role of liraglutide in the relationship between the GLP-1 axis and female reproduction remains to be elucidated, in particular the GLP-1 impact on endometrial quality and receptivity.

\section{Declaration of interest}

The authors declare that there is no conflict of interest that could be perceived as prejudicing the impartiality of this study.

\section{Funding}

The study was supported by the grant number 20140031 of the University Medical Center, Ljubljana. Slovenia.

\section{Author contribution statement}

$\mathrm{V}$ Š: protocol development and data collection, manuscript writing. $\mathrm{M} \mathrm{J}$ : protocol development and data collection, manuscript writing and editing. A J: protocol development, project supervision and manuscript editing. E V B: protocol development, project supervision and manuscript editing.

\section{Acknowledgements}

The authors thank Ivan Verdenik for statistical analysis and most helpful discussions and advice. The authors' special thanks go to the Embryology team for their persistent and accurate IVF laboratory work and appreciate the kind assistance of nurses at the Department of Human Reproduction and Department of Endocrinology, Diabetes and Metabolic Diseases.

\section{References}

1 Yildiz BO, Bozdag G, Yapici Z, Esinler I \& Yarali H. Prevalence, phenotype and cardiometabolic risk of polycystic ovary syndrome under different diagnostic criteria. Human Reproduction 201227 3067-3073. (https://doi.org/10.1093/humrep/des232)

2 Rotterdam ESHRE/ASRM-Sponsored PCOS Consensus Workshop Group. Revised 2003 consensus on diagnostic criteria and long-term 
health risks related to polycystic ovary syndrome. Fertility and Sterility 200481 19-25. (https://doi.org/10.1016/j.fertnstert.2003.10.004)

3 Yildiz BO, Knochenhauer ES \& Azziz R. Impact of obesity on the risk for polycystic ovary syndrome. Journal of Clinical Endocrinology and Metabolism 200793 162-168. (https://doi.org/10.1210/jc.2007-1834)

4 Luke B, Brown MB, Missmer SA, Bukulmez O, Leach R \& Stern JE. The effect of increasing obesity on the response to and outcome of assisted reproductive technology: a national study. Society for Assisted Reproductive Technology writing group. Fertility and Sterility 201196 820-825. (https://doi.org/10.1016/j.fertnstert.2011.07.1100)

5 Luke B. Adverse effects of female obesity and interaction with race on reproductive potential. Fertility and Sterility 2017107 868-877. (https://doi.org/10.1016/j.fertnstert.2017.02.114)

6 Cui N, Wang H, Wang W, Zhang J, Xu Y, Jiang L, Yang A \& Hao G. Impact of body mass index on outcomes of in vitro fertilization/ intracytoplasmic sperm injection among polycystic ovarian syndrome patient. Journal of Assisted Reproduction and Genetics 2017 34 61-70. (https://doi.org/10.1007/s10815-016-0830-1)

7 Moran L, Tsagareli V, Norman R \& Noakes M. Diet and IVF pilot study: short-term weight loss improves pregnancy rates in overweight/obese women undertaking IVF. Australian and New Zealand Journal of Obstetrics and Gynaecology 201151 455-459. (https://doi.org/10.1111/j.1479-828X.2011.01343.x)

8 Sim KA, Dezarnaulds GM, Denyer GS, Skilton MR \& Caterson ID. Weight loss improves reproductive outcomes for obese women undergoing assisted reproductive technology: a randomised controlled trial. Clinical Obesity 201414 792-805.

9 Espinós JJ, Polo A, Sánchez-Hernández J, Bordas R, Pares P, Martínez O \& Calaf J. Weight decrease improves live birth rates in obese women undergoing IVF: a pilot study. Reproductive BioMedicine Online 201735 417-424. (https://doi.org/10.1016/j. rbmo.2017.06.019)

10 Palomba S, Falbo A, Valli B, Morini D, Villani MT, Nicoli A \& La Sala GB. Physical activity before IVF and ICSI cycles in infertile obese women: an observational cohort study. Reproductive BioMedicine Online 201429 72-79. (https://doi.org/10.1016/j.rbmo.2014.03.006)

11 Sim KA, Partridge SR \& Sainsbury A. Does weight loss in overweight or obese women improve fertility treatment outcomes? A systematic review. Obesity Reviews 201415 839-850. (https://doi.org/10.1111/ obr.12217)

12 Wekker V, Van Dammen L, Van Oers AM, Mutsaerts MAQ, Painter RC, Zwinderman AH, Groen H, Van De Beek C, Muller Kobold AC, Land JA et al. Effect of a lifestyle intervention in obese infertile women on cardiometabolic health and quality of life: results of a randomised controlled trial. In Abstracts of the 33rd Annual Meeting of ESHRE. Geneva, Switzerland, 2-5 July 2017.

13 Marchi J, Berg M, Dencker A, Olander EK \& Begley C. Risks associated with obesity in pregnancy for the mother and baby: a systematic review of reviews. Obesity Reviews 201516 621-638. (https://doi. org/10.1111/obr.12288)

14 Thessaloniki ESHRE/ASRM-Sponsored PCOS Consensus Workshop Group. Consensus on infertility treatment related to polycystic ovary syndrome. Human Reproduction 200823 462-477. (https://doi. org/10.1093/humrep/dem426)

15 ESE PCOS Special Interest Group. The polycystic ovary syndrome: a position statement from the European Society of Endocrinology. European Journal of Endocrinology 2014171 P1-P29. (https://doi. org/10.1530/EJE-14-0253)

16 Palomba S, Falbo A \& La Sala. Effects of metformin in women with polycystic ovary syndrome treated with gonadotrophins for in vitro fertilisation and intracytoplasmic sperm injection cycles: a systematic review and meta-analysis of randomised controlled trials. Reproductive BioMedicine Online 2013120 267-276. (https://doi. org/10.1111/1471-0528.12070)

17 Morley LC, Tang TMH, Balen AH \& Royal College of Obstetricians and Gynaecologistws. Metformin therapy for the management of infertility in women with polycystic ovary syndrome. British Journal of Obstetrics and Gynaecology: An International Journal of Obstetrics and Gynaecology 2017124 306-313. (https://doi.org/10.1111/14710528.14371)

18 Nylander M, Frøssing S, Kistorp C, Faber J \& Skouby OS. Liraglutide in polycystic ovary syndrome: a randomized trial, investigating effects on thrombogenic potential. Endocrine Connections 20176 89-99. (https://doi.org/10.1530/EC-16-0113)

19 Frossing S, Nylander, Elizaveta Chabanova, Frystyk J, Holst JJ, Kistorp C, Skouby OS \& Faber J. Effect of liraglutide on ectopic fat in polycystic ovary syndrome: a randomized clinical trial. Diabetes, Obesity and Metabolism 201820 215-218. (https://doi.org/10.1111/ dom.13053)

20 Jensterle Sever M, Kocjan T, Pfeifer M, Kravos NA \& Janez A. Shortterm combined treatment with liraglutide and metformin leads to significant weight loss in obese women with polycystic ovary syndrome and previous poor response to metformin. European Journal of Endocrinology 2014170 451-459. (https://doi.org/10.1530/EJE-130797)

21 Abu-Hamdah R, Rabiee A, Meneilly GS, Shannon RP, Andersen DK \& Elahi D. Clinical review: the extrapancreatic effects of glucagon-like peptide-1 and related peptides. Journal of Clinical Endocrinology and Metabolism 200994 1843-1852. (https://doi.org/10.1210/jc.20081296)

22 Woods SC \& D'Alessio DA. Central control of body weight and appetite. Journal of Clinical Endocrinology and Metabolism 200893 37-50. (https://doi.org/10.1210/jc.2008-1630)

23 Outeiriño-Iglesias V, Romaní-Pérez M, González-Matías LC, Vigo E \& Mallo F. GLP-1 increases pre-ovulatory LH source and the number of mature follicles as well as synchronizing the onset of puberty in female rats. Endocrinology 2015156 4226-4237.

24 Barber TM, Wass JA, McCarthy MI \& Franks S. Metabolic characteristics of women with polycystic ovaries and oligoamenorrhoea but normal androgen levels: implications for the management of polycystic ovary syndrome. Clinical endocrinology 200766 513-517.

25 Expert Committee on the Diagnosis and Classification of Diabetes Mellitus. Follow-up report on the diagnosis of diabetes mellitus. Diabetes Care 200326 3160-3167.

26 Ryo M, Maeda K, Onda T, Katashima M, Okumiya A, Nihida M, Yamaguchi T, Funahashi T, Matsuzawa Y, Nakamura T et al. A new simple method for the measurement of visceral fat accumulation by bioelectrical impedance. Diabetes Care 200528 451-453. (https://doi. org/10.2337/diacare.28.2.451)

27 Matthews DR, Hosker JP, Rudenski AS, Naylor BA, Treacher DF $\&$ Turner RC. Homeostasis model assessment: insulin resistance and b-cell function from fasting plasma glucose and insulin concentrations in man. Diabetologia 198528 412-419. (https://doi. org/10.1007/BF00280883)

28 Virant-Klun I, Tomazevic T, Bacer-Kermavner L, Mivsek J, Valentincic-Gruden B \& Meden-Vrtovec H. Successful freezing and thawing of blastocysts cultured in sequential media using a modified method. Fertility and Sterility 200379 1428-1433. (https://doi. org/10.1016/S0015-0282(03)00395-9)

29 Gardner DK, Lane M, Stevens J, Schlenker T \& Schoolcraft WB. Blastocyst score affects implantation and pregnancy outcome: towards a single blastocyst transfer. Fertility and Sterility $2000 \mathbf{7 3}$ 1155-1158. (https://doi.org/10.1016/S0015-0282(00)00518-5)

30 Nicklas BJ, Penninx BW, Ryan AS, Berman DM, Lynch NA \& Dennis KE. Visceral adipose tissue cutoffs associated with metabolic risk factors for coronary heart disease in women. Diabetes Care 200326 1413-1420. (https://doi.org/10.2337/ diacare.26.5.1413)

31 Mutsaerts MA, van Oers AM, Groen H, Burggraaff JM, Kuchenbecker WK, Perquin DA, Koks CA, van Golde R, Kaaijk EM, Schierbeek JM et al. Randomized trial of a lifestyle program in 
obese infertile women. New England Journal of Medicine 2016374 1942-1953. (https://doi.org/10.1056/NEJMoa1505297)

32 Jensterle M, Goricar K \& Janez A. Metformin as an initial adjunct to low-dose liraglutide enhances the weight-decreasing potential of liraglutide in obese polycystic ovary syndrome: randomized control study. Experimental and Therapeutic Medicine 201611 1194-1200. (https://doi.org/10.3892/etm.2016.3081)

33 Nieuwenhuis-Ruifrok AE, Kuchenbecker WKH, Hoek A, Middleton P $\&$ Norman RJ. Insulin sensitizing drugs for weight loss in women of reproductive age who are overweight or obese: systematic review and meta-analysis. Human Reproduction Update 200915 57-68 (https:// doi.org/10.1093/humupd/dmn043)

34 Tso LO, Costello MF, Albuquerque LE, Andriolo RB. Freitas V $\&$ Macedo CR. Metformin treatment before and during IVF or ICSI in women with polycystic ovary syndrome. Cochrane Database of Systematic Reviews 201411 CD006105. (https://doi. org/10.1002/14651858.CD006105.pub3)

35 Liu X, Zhang Y, Zheng SY, Lin R, Xie YJ, Chen H, Zheng YX, Liu E, Chen L, Yan JH et al. Efficacy of exenatide on weight loss, metabolic parameters and pregnancy in overweight/obese polycystic ovary syndrome. Clinical Endocrinology 201787 767-774. (https://doi. org $/ 10.1111 /$ cen.13454)

36 Palomba S, Giallauria F, Falbo A, Russo T, Oppedisano R, Tolino A, Colao A, Vigorito C, Zullo F \& Orio F. Structured exercise training programme versus hypocaloric hyperproteic diet in obese polycystic ovary syndrome patients with anovulatory infertility: a 24-week pilot study. Human Reproduction 200823 642-650. (https://doi. org/10.1093/humrep/dem391)

37 Huber-Buchholz MM, Carey DG \& Norman RJ. Restoration of reproductive potential by lifestyle modification in obese polycystic ovary syndrome: role of insulin sensitivity and luteinizing hormone. Journal of Clinical Endocrinology and Metabolism 199984 1470-1474.

38 Elkind-Hirsch K, Marrioneaux O, Bhushan M, Vernor D \& Bhushan R. Comparison of single and combined treatment with exenatide and metformin on menstrual cyclicity in overweight women with polycystic ovary syndrome. Journal of Clinical Endocrinology and Metabolism 200893 2670-2678. (https://doi. org/10.1210/jc.2008-0115)

39 Teede Hutchison SK \& Zoungas S The management of insulin resistance in polycystic ovary syndrome. Trends in Endocrinology and Metabolism 200718 273-279. (https://doi.org/10.1016/j. tem.2007.08.001)

40 Beak SA, Heath MH, Small CJ, Morgan DGA, Ghatei MA, Taylor AD, Buckingham JC, Bloom SR \& Smith DM. Glucagon-like peptide-1 stimulates luteinizing hormone-releasing hormone secretion in a rodent hypothalamic neuronal cell line. Journal of Clinical Investigation 1998101 1334-1341. (https://doi.org/10.1172/JCI610)

41 Outeiriño-Iglesias V, Romaní-Pérez M, González-Matías LC, Vigo E \& Mallo F. GLP-1 increases pre-ovulatory LH source and the number of mature follicles, as well as synchronizing the onset of puberty in female rats. Endocrinology 2015156 4226-4237. (https://doi. org/10.1210/en.2014-1978)

42 Jeibmann A, Zahedi S, Simoni M, Nieschlag E \& Byrne MM. Glucagon-like peptide-1 reduces the pulsatile component of testosterone secretion in healthy males. European Journal of Clinical Investigation 200535 565-572. (https://doi.org/10.1111/j.13652362.2005.01542.x)

43 Cunningham MJ, Clifton DK \& Steiner RA. Leptin's actions on the reproductive axis: perspectives and mechanisms. Biology of Reproduction 199960 616-622.

44 Maclusky NJ, Cook S, Scrocchi L, Shin J, Kim J, Vaccarino F, Asa SL $\&$ Drucker DL. Neuroendocrine function and response to stress in mice with complete disruption of glucagon-like peptide-1 receptor signaling. Endocrinology 20002 752-762. (https://doi.org/10.1210/ endo.141.2.7326)

45 Garris DR. Diabetes (db/db) mutation-induced endometrial epithelial lipoapoptosis: ultrastructural and cytochemical analysis of reproductive tract atrophy. Reproductive Biology and Endocrinology 20053 15-26. (https://doi.org/10.1186/1477-7827-3-15)

46 Artunc-Ulkumen B, Pala HG, Pala EE, Yavasoglu A, Yigitturk G \& Erbas $\mathrm{O}$. Exenatide improves ovarian and endometrial injury and preserves ovarian reserve in streptozocin induced diabetic rats. Gynecological Endocrinology 201531 196-201. (https://doi.org/10.310 9/09513590.2014.975686)

47 Bethel MA, Patel RA, Merrill P, Lokhnygina Y, Buse JB, Mentz RJ, Pagidipati NJ, Chan JC, Gustavson SM, Iqbal N et al. Cardiovascular outcomes with glucagon-like peptide-1 receptor agonists in patients with type 2 diabetes: a meta-analysis. Lancet Diabetes and Endocrinology 20186 105-113. (https://doi.org/10.1016/S22138587(17)30412-6)

48 Greco D. Normal pregnancy outcome after first-trimester exposure to liraglutide in a woman with Type 2 diabetes. Diabetic Medicine 2015 32 29-30. (https://doi.org/10.1111/dme.12726)

49 Yang Q \& Wang F. Successful pregnancy after improving insulin resistance with the glucagon-like peptide- 1 analogue in a woman with polycystic ovary syndrome: a case report and review of the literature. Gynecologic and Obstetric Investigation 201681 477-480. (https://doi.org/10.1159/000446951)

Received 27 February 2018

Revised version received 17 April 2018

Accepted 27 April 2018 